\title{
Análisis cualitativo de tópicos vinculados a la calidad de vida en personas con discapacidad
}

\author{
Qualitative analysis of topics related to the quality of life \\ of people with disabilities
}

María Victoria Martín Cilleros ${ }^{1}$

Maria Cruz Sánchez Gómez ${ }^{1}$

${ }^{1}$ Departamiento de Didáctica, Organización y Métodos de Investigación, Facultad de Educación, Universidad de Salamanca. Paseo de Canalejas 169. 37008 Salamanca España. viquimc@usal.es

\begin{abstract}
Quality of life is a concept that reflects the subjective perception of individuals in relation to their degree of satisfaction with their living conditions. It is a concept that involves putting the individual at the forefront, talking about the quality of the professionals involved and influencing the development of programs and the provision of services. The article reflects the opinions that different agents have in relation to disability on given topics related to the model of quality of life such as: the relationship between different agents; the necessary training of professionals in the field of health and/or disability; and the existence of the presence of the participation in society of people with disabilities. Using qualitative methodology, the arguments of the participants in these three issues are discussed. The verbiage, the conceptual map and the analysis of content, performed after the encoding of information, made it possible to detect the perceived needs and satisfaction with the care of the different services. The profile of the qualified professional within the scope of disability is outlined. Proposals for the future are contained in the conclusions, ensuring the improvement of the quality of life of people with disabilities.
\end{abstract}

Key words Inclusion, Disability, Support, Quality of life, Qualitative research
Resumen La calidad de vida es un concepto que refleja la percepción subjetiva de la persona en relación al grado de satisfacción con sus condiciones de vida. Es un concepto que implica poner en primer plano a la persona, hablar sobre la calidad de los profesionales implicados e influye en el desarrollo de programas y la provisión de los servicios. El artículo refleja la opinión que tienen diferentes agentes relacionados con la discapacidad sobre determinados tópicos vinculados al modelo de calidad de vida como son: relación entre diferentes agentes, la formación necesaria en los profesionales del ámbito de la salud y/o de la discapacidad $y$ el paso de la presencia a la participación en la sociedad de las personas con discapacidad. A través de una metodológica cualitativa, se analizan las argumentaciones de los participantes en estos tres temas. La nube de palabras, el mapa conceptual y el análisis de contenido, realizado tras la codificación de la información, permitieron conocer las necesidades percibidas y la satisfacción con la atención de los diferentes servicios. Se propone el perfil del profesional idóneo en el ámbito de la discapacidad. En las conclusiones obtenidas se recogen propuestas de futuro, facilitadoras de la mejora de la calidad de vida de las personas con discapacidad.

Palabras-clave Inclusión, Discapacidad, Apoyos, Calidad de vida, Metodología cualitativa 


\section{Introducción}

A lo largo de la historia del ser humano, la discapacidad ha sido abordada desde diferentes modelos, se ha evolucionado de un modelo demonológico a un modelo biopsicosocial, pasando por un modelo médico. Estos modelos se han ido desarrollando de acuerdo a la concepción que la sociedad tenía de la misma; además de por la influencia de otros factores como pueden ser los resultados de la investigación, la preocupación por dar soluciones a las necesidades planteadas por las personas con discapacidad y sus familias, entre otros. En esta evolución un modelo no ha supuesto la eliminación del anterior, al contrario, las diferentes concepciones han ido en paralelo, superponiéndose en el tiempo, situación que ha dado lugar a que convivan en el mismo contexto sociocultural nociones diferentes, presentándose incluso en algunas situaciones creencias que reflejan modelos que se creían haber dejado en el pasado. Puede incluso que utilicemos la terminología que representa la ideología del modelo de apoyos y calidad de vida, modelo que más aceptación e impacto tiene en el ámbito internacional por sus implicaciones prácticas ${ }^{1}$, pero dichas prácticas se realicen desde los principios de un modelo rehabilitador, conviviendo incluso en una misma institución, diferentes actitudes hacia la discapacidad. ¿Cuál es el modelo más deseado por las personas afectadas?:En una época en la que se busca mejorar la calidad de vida de los pacientes, cómo es percibida ésta por dichas personas?

La inclusión plena de la persona con discapacidad en la sociedad da como resultado una mejora en su calidad de vida. La inclusión debe ser considerada como una búsqueda permanente de procedimientos, cada vez más adecuados, para responder a la diversidad, convivir con la diferencia y aprender a aprender de ella ${ }^{2}$. Tiene que ver con el proceso de incrementar y mantener la participación de todas las personas en la sociedad, escuela o comunidad de forma simultánea, procurando disminuir y eliminar todo tipo de procesos que lleven a la exclusión ${ }^{3}$. Consiste por tanto en construir una sociedad viva, que permita el desarrollo, aprendizaje y éxito social de cualquiera de sus ciudadanos ${ }^{4}$. Donde el hecho de ser ciudadano conlleva el derecho de la participación en actividades que integran el universo existencial humano, prerrequisito fundamental para la calidad de vida y promoción de la salud ${ }^{5}$. La ciudadanía sin embargo, es un proceso social establecido, puesto que los derechos no son otor- gados y confeccionados por los estados, sino que existen más como "prácticas sociales relacionales", esto es, como principios éticos o morales que en determinadas "condiciones de lugar, cultura política y participación" se transforman en derechos. En el caso de las personas con discapacidad, las luchas del colectivo son las que han facilitado la formalización de determinados derechos que ya estaban latentes; pudiendo darse también el caso contrario de que determinados derechos subjetivos han sido asumidos formalmente llegando a convertirse en principios, inspiradores de un cambio social ${ }^{6}$.

Por tanto, el éxito de las políticas tendentes a priorizar el modelo de inclusión social de las personas con discapacidad, depende en gran medida del grado de conocimiento, las creencias y las actitudes de la sociedad hacia la discapacidad. Cuanto más escaso sea el conocimiento, las actitudes serán más negativas y las creencias más estigmatizantes?.

Nos encontramos así que las organizaciones, los profesionales que se comprometen con las personas con discapacidad, las familias y los propios interesados forman un conjunto inseparable y clave para el desarrollo de prácticas eficaces que deriven en resultados personales de calidad para las personas con discapacidad. Siendo necesario que nuevas y eficaces políticas públicas favorezcan la redefinición de las organizaciones, potenciando su transformación positiva para mejorar la eficacia y eficiencia en los resultados debido a las buenas prácticas de los profesionales ${ }^{1}$.

Debido a que los enfoques cualitativos están fuertemente relacionados con los deseos, las necesidades, las metas y las promesas de una sociedad democrática; puesto que los investigadores que trabajan en este campo deben asumir un compromiso de ciudadanía con la gente y los problemas que investigan ${ }^{8,9}$ se decidió indagar mediante el análisis de contenido, en la opinión que tenían sobre la inclusión las personas afectadas, familiares y profesionales relacionados en dicho ámbito. Hernández et al..$^{10}$, señalan que el enfoque cualitativo se utiliza cuando se busca comprender la perspectiva de los participantes (individual o en grupo) acerca de los fenómenos que los rodean, profundizar en sus experiencias, perspectivas, opiniones y significados, es decir, la forma en que los participantes perciben la realidad.

En el contexto de una jornada transfronteriza entre Salamanca (España) y Castelo Branco (Portugal), donde se puso en común las prácticas de inclusión que se estaban llevando a cabo en ambos lugares, se analizó el posterior debate 
generado en torno a tres mesas de trabajo: la relación que existe entre los diferentes agentes involucrados en potenciar la inclusión, el camino que queda por recorrer para ir de la presencia de las personas con discapacidad en la sociedad a la participación que lleva a la mejora de la calidad de vida y la formación necesaria para los profesionales que prestan servicios a las personas con discapacidad, como pueden ser profesionales sanitarios o educativos, o aquellos que pueden ser determinantes para avanzar hacia una sociedad más democrática.

\section{Métodología}

\section{Objetivos}

Analizar el debate que se estableció entre los diferentes miembros relacionados con el ámbito de la discapacidad que permitiera recoger las ideas que tenían del estado de la inclusión y por tanto de la calidad de vida, los factores facilitadores de la misma así como las barreras que actualmente encuentran en el día a día. El debate a su vez permitió la reflexión sobre propuestas de futuro que favorezcan el avance hacia modelos basados en los apoyos comunitarios y participación plena de las personas con discapacidad.

\section{Participantes}

En el marco de movilidad vinculada con los convenios de cooperación con universidades extranjeras y otras instituciones de enseñanza superior, se llevó a cabo una estancia en la universidad de Salamanca de docentes pertenecientes al Instituto Politécnico de Educación de Castelo Branco. Entre otras actividades, se realizó una jornada dirigida a la exposición y posterior debate de prácticas de inclusión aplicadas a la discapacidad en ambos países.

A dicha actividad acudieron personas con algún tipo de discapacidad de orden física, motora, sensorial, mental y/o múltiple, familiares, técnicos que prestan sus servicios en las asociaciones así como profesionales del ámbito educativo, estando representado en éste último, especialistas de enseñanzas medias y superiores. Igualmente se contó con representación de futuros profesionales. Se disponía por tanto, de una representatividad muestral discursiva de acuerdo a los diferentes colectivos, siendo un total de veintitrés personas (Figura 1) las que participan en el debate estructurado en tres mesas de trabajo.

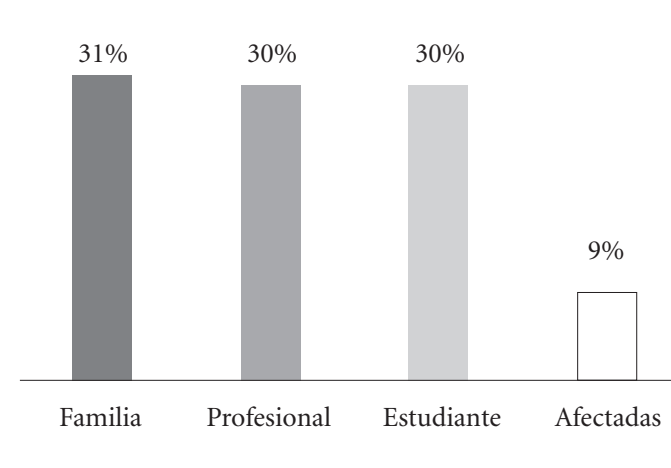

Figura 1. Porcentaje de representación de los participantes.

Entre las asociaciones representadas se encontraban fibromialgia y síndrome de fatiga crónica, problemas visuales, problemas auditivos, esclerosis múltiple, parálisis cerebral, hiperactividad, autismo, síndrome de Down, discapacidad intelectual y una asociación de equinoterapia que ofrece sus servicios a todo tipo de discapacidad.

\section{Metodología}

La universidad de Salamanca, entre una de las responsabilidades que tiene con la sociedad, está el promover el compromiso responsable y la participación activa en la defensa y la lucha por la diversidad y los derechos humanos.

En esta situación cedió sus espacios para el desarrollo de la jornada y utilización de sus materiales, que permitieran la conexión vía internet con profesionales de Portugal.

La jornada comenzó con varias charlas sobre políticas educativas y buenas prácticas de inclusión en Portugal así como el uso de las tecnologías de la información y comunicación aplicadas a la discapacidad, conectando online con la Asociación Portuguesa de Padres y Amigos de Personas con Discapacidad Intelectual (APPACDM). Esta conexión permitió un intercambio de ideas a nivel internacional y tener una base para el debate. Le siguió una pausa informal que permitió generar un clima distendido y de conocimiento entre los profesionales.

Tras la pausa, y bajo un enfoque cualitativo, se establecieron tres mesas de trabajo donde se debatieron los siguientes tópicos sobre discapacidad: la relación entre los diferentes agentes, es decir entre la familia o afectados, los profesionales y las instituciones; la formación de los profe- 
sionales y el paso de la presencia a la participación en la sociedad por parte de las personas con discapacidad.

Todos los participantes abordaron las diferentes temáticas, rotando en grupos de una a otra al pasar un tiempo determinado, aproximadamente una hora. Se estructuraron los grupos de forma que quedaran equilibrados en estratos según tipo de participante. Cada grupo de trabajo fue dirigido por la misma persona, que tenía la función de moderador, lo cual aseguró la fiabilidad en la conducción de los tópicos tratados. En todos los grupos se abrió el debate con una pregunta abierta.

La puesta en común en las diferentes mesas se desarrolló con fluidez, y en un clima muy participativo. El contexto facilitador previo a las mismas, unido a que las dinámicas de los grupos se llevaron a cabo de forma abierta y no directiva, fomentó que los participantes hablaran con libertad, expresando sus ideas de forma individual e interactiva. El contenido se grabó en audio, previa autorización de los participantes.

Mediante estas entrevistas grupales, se obtiene información sobre un tema focalizado en un contexto social, donde las personas pueden considerar sus visiones en interacción con los demás. Esta estrategia metodológica ha permitido acceder a la realidad, sin categorizarla previamente, a través de lo expresado por los sujetos de forma espontánea en contextos naturales. Toda la producción discursiva, libre y espontáneamente producida, se considera relevante para el objeto de estudio. Ningún aspecto de las narraciones, en el que ha sido posible explorar significados, creencias y actitudes, ha quedado fuera del foco de estudio.

\section{Transcripción y análisis de datos}

El análisis consiste en transformar datos textuales no estructurados en estructurados para poder interpretarlos. En este trabajo se ha utilizado la técnica del análisis cualitativo de contenido siguiendo una serie de etapas que constituyen un proceso analítico básico o común a la mayoría de las investigaciones que trabajan con este tipo de datos, basándose en el esquema general de Miles et al. ${ }^{11}$ (Figura 2): a) reducción de datos; b) disposición y transformación de datos; y c) obtención de resultados y verificación de conclusiones. En investigación cualitativa estas fases se pueden superponer, ya que el diseño cualitativo es emergente $^{12,13}$, y se elabora a medida que avanza la investigación. La situación que genera el problema da lugar a un cuestionamiento continuado, y a una reformulación constante, en función de la incorporación de nuevos datos.

Una vez transcritas las entrevistas grupales se procedió a la categorización y codificación de la información recogida. Se desarrolló el mapa conceptual de categorías en función de los objetivos del estudio y del protocolo de preguntas del que se disponía para guiar el debate.

El instrumento de análisis ha sido la organización de las narraciones en ejes temáticos ordenados de forma jerárquica. Esta ordenación de las ideas relevantes o ejes temáticos constituye el sistema de categorías que han permitido el estudio sistemático del contenido de las mismas.

Para facilitar el trabajo de análisis, se ha utilizado el programa informático NVIVO 10, mediante el cual y siguiendo el modelo anteriormente presentado, se ha realizado el mapa conceptual de la categorización basada en las opiniones manifestadas por los colectivos relacionados con la discapacidad organizada en tres metacategorías o ejes principales: formación de los profesionales, relación (entre profesionales) y participación, y once categorías y subcategorías (Figura 3).

\section{Resultados}

En primer lugar se hizo un análisis de frecuencia de palabras en las entrevistas grupales. El fin de esta tarea es explorar los términos más mencionados con el objetivo de averiguar cuáles son los más relevantes. Para el conteo de la frecuencia de palabras se establecieron como criterios de selección las cincuenta más frecuentes. Se depuró la lista en cuatro ocasiones descartando las palabras denominadas vacías o sin contenido. En la siguiente nube de palabras (Figura 4) se ven las once palabras más representativas (con mayor carga porcentual).

La nube de palabras, el mapa conceptual y el análisis de contenido realizado tras la codificación de la información, reflejan ideas extraídas en cada una de las mesas de trabajo que dan lugar a los resultados que se detallan a continuación.

\section{La relación entre los diferentes agentes relacionados en el ámbito de la discapacidad}

En esta mesa se preguntó a los participantes qué aportaciones hace cada uno de los agentes vinculados a la discapacidad (familia, profesionales, instituciones) a la calidad de vida de la persona afectada y sus familias, cómo es la relación entre ellos y cómo se puede mejorar. 


\section{Análisis de datos}

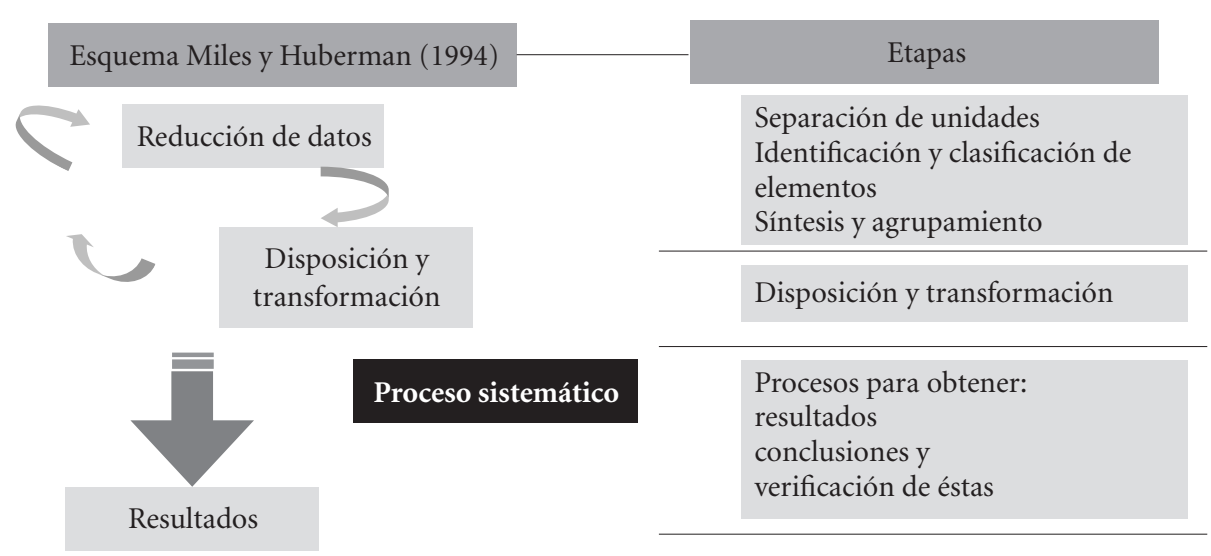

Figura 2. Esquema de proceso analítico de los datos según Miles et al. ${ }^{11}$.

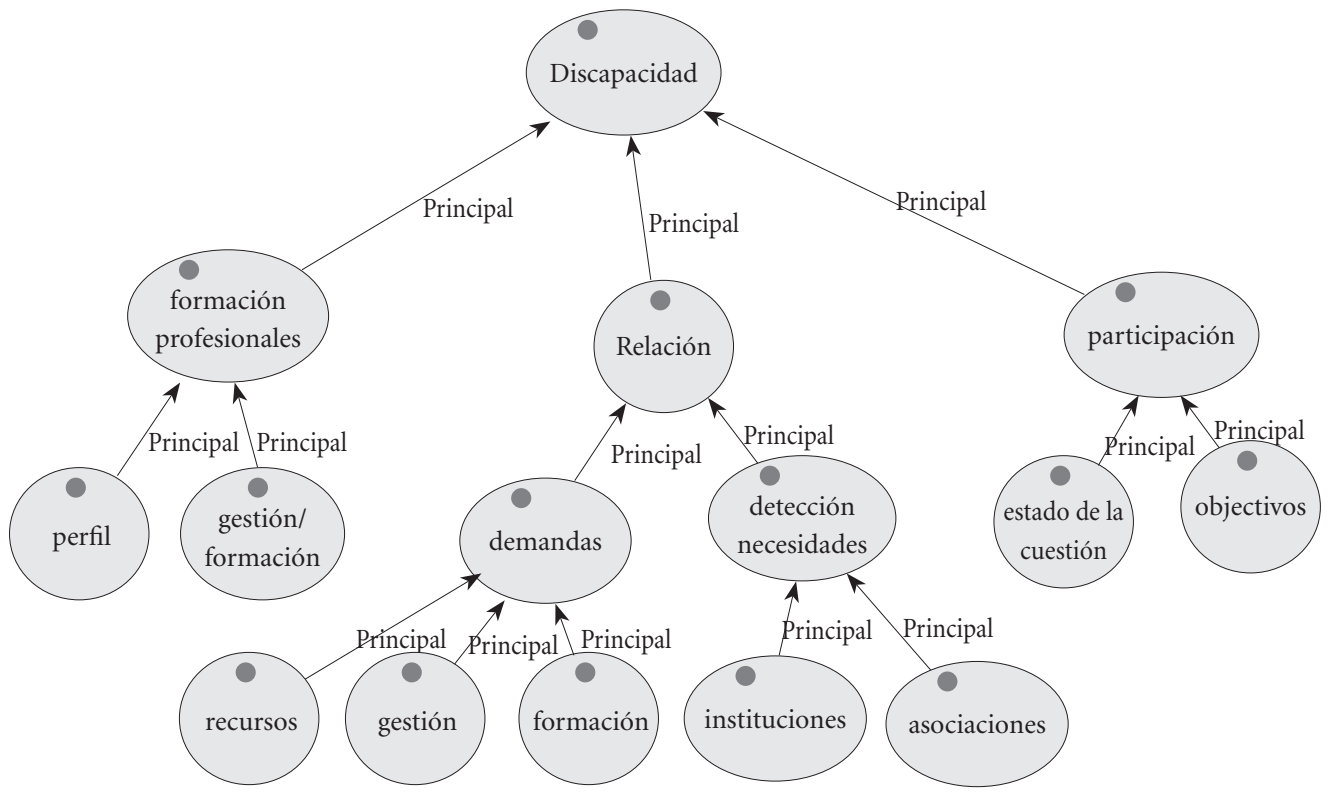

Figura 3. Mapa conceptual del debate.

Todos los participantes están de acuerdo en la falta de interrelación entre ellos. Se carece de una meta común, cada colectivo tiene unos intereses diferentes, que hacen que los esfuerzos se diluyan. Como expresaba un padre esto es una silla de tres patas, para que esté bien asentada tiene que haber una implicación y una comunicación más cercana entre lo que es el problema real, es decir, las familias, lo que nos da la sociedad para paliar ciertos problemas que son los educadores y luego los profesionales que apoyan a todo lo demás...Quiero decir que no podemos tirar cada uno del mismo problema pero para lados diferentes. Se aprecia además una falta de interdisciplinaridad que va en detrimento de la 


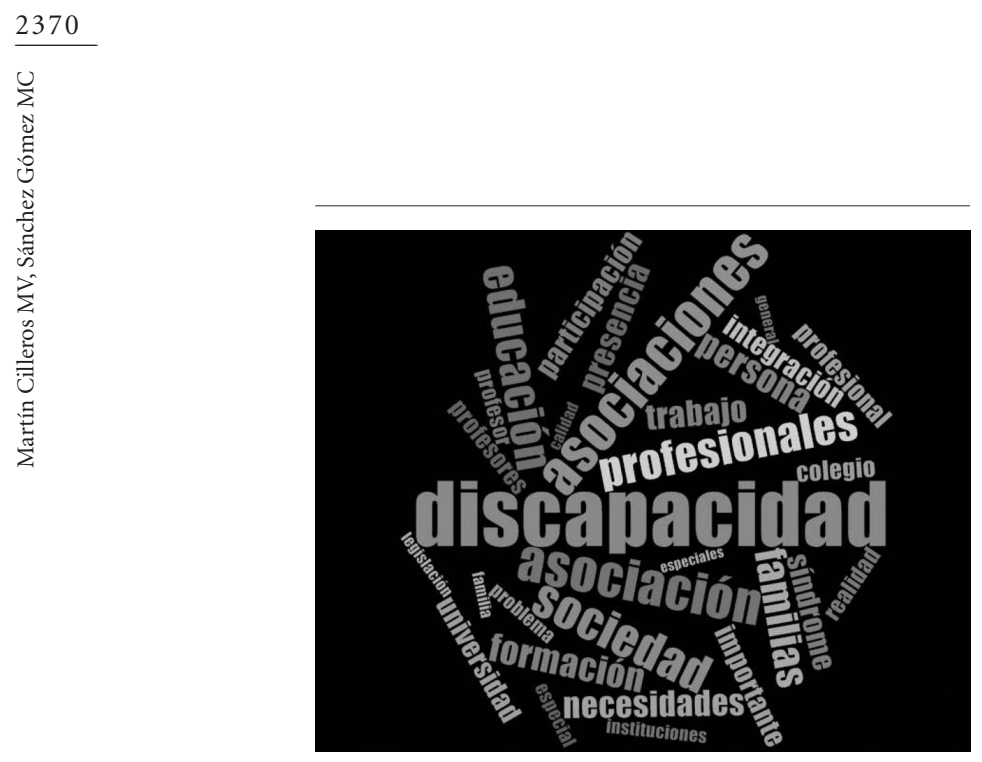

Figura 4. Nube de palabras.

calidad de vida de la persona. Cada profesional se limita a proporcionar sus servicios desde su área, tratándola por parcelas y no viendo la globalidad, como ejemplo un profesional educativo expresaba ellos lo hacen con toda su buena intención, pero solamente tienen información médica, de mi trocito. Vamos a ver si consigo que recupere un poquito, un poquito de visión y para eso tiene que estar siete horas con parche. Todo el enfrentamiento que suponga entre papá, mamá, hermanos, en el cole, lo que le supongan al niño... No lo saben, porque nadie les ha dicho que eso puede ser importante. Indican que dentro de las propias instituciones y asociaciones debería fomentarse la coordinación entre los diferentes profesionales y el trabajo en equipo, incluyendo en dicho equipo en la medida de lo posible, a las personas con discapacidad, dándole voz y escuchando la importancia de abordar los problemas desde su punto de vista.

Se sigue con una falta de horizontalidad en las instituciones (educativas, sanitarias...), donde las familias asumen, o se ven obligadas a asumir, un rol de inferioridad. Incluso a veces sienten que no son escuchadas los orientadores, éstos que son los que evalúan a tu hijo casi sin oír, sin oírte, sin... ¿sabes?, o incluso amenazadas con relegar a la persona con discapacidad a un servicio menos inclusivo.

Otro aspecto que reflejan, por la situación económica que se vive en ese momento en España, es la ausencia de recursos. Esta carencia es vivida por parte de las asociaciones como un retroceso en aspectos conseguidos años atrás la asociación no se mantiene, yo entiendo que tiene que partir de abajo, pero volvemos a los tiempos de la mendicidad de 'entre todos' el de la tele, a la buena voluntad de las personas. Esto no son derechos esto son beneficiencias. Consideran que las asociaciones deben ser el puente entre la familia y la institución, pero debido a una cada vez mayor especialización de las mismas, enfocadas a una problemática muy concreta, hace que las fuerzas se dividan y consecuentemente los recursos a repartir. Consideran que aquellas asociaciones que más recursos tienen, sobre todo humanos, más crecen, puesto que están más preparadas para presentar a la administración la cantidad de trámites necesarios para recibir las subvenciones tal y como decía un familiar los recursos que cada vez se nos dificultan a propósito, y estoy convencido que a propósito, o sea, para pedir cualquier subvención tienes que presentar unos 'tochos' justificativos $y$ de proyectos así, y eso en asociaciones con buenos recursos, con buenos profesionales, ... pues es más sencillo que para nosotros que es complicadísimo, complicadísimo principalmente porque no somos personas..., somos padres, no estamos preparados.

Los recortes, llevados a cabo en el ámbito de la salud y en las ayudas por situación de dependencia, hacen que las familias sientan que no se da cobertura a sus necesidades, o es necesario mucho tiempo y esfuerzo por parte de los representantes (familia y asociación) para que sean escuchadas Los profesionales y familias tenemos que ir tirando del carro de la administración... tienes que ir de la mano, tirando y explicando las necesidades a la administración... y negociar con ellos 5 ó 6 años. Buscando ellos mismos las soluciones para que sean canalizadas mínimamente, como indicaban de las administraciones, yo como consejo después de que ya llevo 20 años ya no sé cuántos, ya no espero nada, sí pedirles imaginación mira dinero no os puedo pedir pero, imaginación sí, y además como ya sé de sobra que hay que llevarle la solución, ya le llevamos la solución. Estos recortes y falta de recursos, son vividos en la institución educativa como un freno a la inclusión. Debido al aumento de ratio de alumnos por aula y la escasez de apoyos en los colegios ordinarios hace que la presencia de una persona con discapacidad sea considerada como una traba, tanto para el resto de alumnos como para los profesores. Por otra parte se han eliminado del currículo materias que daban lugar a espacios que permitían a la persona con discapacidad aumentar su autoestima. Se muestra un extracto de un técnico en educación sobre dicha opinión ...y la música, y la educación física, y la psicomotricidad, y las manualidades, la plástica... Es decir, pero ¿por qué? Porque todo eso también como no se hace, como eso no importa... Vas quitando a lo único que estos niños podían destacar, incorporarse. 


\section{La formación de los profesionales}

Aunque este tema fue tratado de manera específica en una de las mesas, se abordó de forma transversal en las demás. En esta mesa se debatió la formación que poseen los profesionales en ejercicio, así como la necesaria para los futuros profesionales, definiendo el perfil ideal que debería de tener el profesional que dedica sus servicios a las personas con discapacidad o por su situación laboral tiene, o puede tener, contacto con ellas.

En general se observa una escasa formación de los profesionales en activo en diferentes aspectos como son, saber detectar a tiempo un problema para comenzar cuanto antes la intervención. De ese modo se evitaría encontrarse con situaciones como las expresadas por una madre Bueno mujer, pero si no camina ya caminará.... Digo vale, pero es que prefiero que la vean y que me digáis que a la niña no le pasa nada y me voy para mi casa tan contenta, pero si le pasa quiero empezar ya. Tanto los profesionales como los familiares están de acuerdo que es necesario saber transmitir la información, sobre todo por los profesionales del ámbito de la salud, priorizando la forma de dar la primera noticia, asintiendo de forma absoluta con la opinión de un profesional que decía Aunque sea una conversación igual de cinco minutos, los psicólogos sabéis que el impacto de cómo te llega la primera noticia cambia radicalmente el curso de las cosas, porque te da energía o te la quita, te hace sentir culpable o te hunde, o te... Me ha caído una losa encima o me están diciendo que tengo complicaciones pero me están dando, abriendo puertas. Además deberían disponer de información que no se centre única y exclusivamente en su área de especialización, ofreciendo información de posibles recursos humanos o materiales, que permitan a la familia o persona con discapacidad avanzar con mayor fluidez hacia una normalización de la situación o adquisición de los apoyos necesarios para ello, como expresaban algunos técnicos otras veces las familias no saben cómo llegar a las asociaciones... Cuáles son los apoyos que puede tener, qué puedo buscar. Esta información es considerada primordial en los inicios, en los que se encuentran en un proceso de duelo, aturdidos por la nueva situación o por la sospecha de un problema, y no saben dónde acudir. Como exponía una madre refiriéndose a un centro de evaluación e intervención (centro base) dirigido a niños de 0 a 3 años, que descubrió que existía, próximo a su zona de residencia Yo por ejemplo, el centro base, viviendo aquí... yo no lo conocía, no sabía ni que existía. Y mi hija fue casi con 3 años al centro base. Y que creo que si eso lo hubiéramos conocido... Y, por ejemplo, el médico, o alguien de la guardería, o alguien nos hubiera dicho: "eso existe", yo hubiera ido, claro, como fui, y haciendo referencia a ese periodo de peregrinación, que tiene lugar en la mayor parte de las familias, acudiendo allá donde creen que puedan ofrecerles información, yo que ir a Burgos, que tampoco nadie me dijo que alli había un centro de referencia de enfermedades raras... que cuando lo supe para allá que fui....

Con el fin de mejorar la situación anterior, se considera necesario que a los futuros profesionales se les debe proporcionar, independientemente de su elección académica, una formación sobre la diversidad, que permita sensibilizar y eliminar barreras mentales, siendo esta formación más amplia y específica en determinados sectores sanitarios y sociales. De hecho consideran que en la gran mayoría de los profesionales dedicados a la discapacidad se nota una sensibilidad hacia dicho colectivo en su labor diaria las personas que trabajan en asociaciones a lo mejor por su perfil o por el hecho de ir directamente a trabajar en ese entorno tienen una formación que tiene más sensibilidad hacia la diversidad humana.

Todos los participantes resaltaban el hecho de una necesaria vinculación entre asociaciones y universidad que permitiera partir de la práctica para obtener y vincular la teoría. A pesar de que en el pasado esta vinculación ha existido, ahora se limita a la visita por parte de los alumnos a título particular con el fin de poder desarrollar sus obligatorios trabajos de fin de grado o máster. Estos encuentros además son poco beneficiosos para ambas partes, como indicaban profesionales de las asociaciones ... Vienen con cuatro cositas que han leido, una idea muy clara y muy pequeña de lo que quieren hacer y te piden esto puntualmente. Asi es muy difícil, porque realmente lo único que estás haciendo es echarles un cable para que presenten el trabajo, pero ni siquiera puedes plantear vamos, ¿queréis hacer un trabajo sobre ceguera? Pues vamos a ver qué temas, cómo lo trabajamos... De manera que al final el trabajo que hagáis sirva para algo a vosotros, a nosotros, a los usuarios y a todo el mundo.

Los profesionales recién titulados encuentran un gran abismo entre la realidad y la formación recibida. Aunque reconocen que es imposible adquirir conocimiento de todas y cada una de las enfermedades y sus necesidades, sí consideran necesario una formación general que después será necesario ampliar. Dicha ampliación debería llevarse a cabo a través de una formación en acción 
dentro de las propias asociaciones, desarrollando y apoyando buenas prácticas asesoradas por expertos. Un profesional expuso una propuesta de futuro que fue ampliamente acogida por el grupo como es, desarrollar acciones formativas en horizontal, independiente de la categoría laboral, que fomentara la coordinación y trabajo en equipo en esos planteamientos de buenas prácticas, a mí me parece que aunque no conozco ninguna experiencia en ese sentido, deberían de estar por igual las familias y distintos perfiles profesionales, porque cuando se tienden hacer estas cosas tiende a ser solo para no sé qué perfil, solo para... y yo creo que lo que más enriquece es que pudieran estar las familias también, y los profesionales.

Finalmente se llegó al perfil del profesional ideal, definiendo que además de saber hacer, debe tener cualidades para trabajar en equipo y transmitir sus conocimientos, debería ser autodidacta, empático y estar motivado con su trabajo. La gran mayoría del grupo valora más ser una persona polivalente que un experto te das cuenta que tanto los profesores que vas a orientar como las familias pues está bien para ellos saber que tú tienes una buena formación, pero tú te comunicas bien, te pones a nivel, acoges, estás dispuesto a aceptar una crítica o una sugerencia eso es lo que realmente es valioso, y creo que la separación que ha habido entre la universidad, la formación y la realidad, las demandas de los profesionales y de las familias, viene de que la universidad, yo creo que se ha quedado un poco en los expertos y ya no hace falta expertos.

En el debate se expone que a la hora de actuar, un profesional debe tener clara la necesidad que tiene la persona en particular y tiene que tener una concienciación sobre el tema, en este caso sobre la discapacidad. Por ello es importante saber y conocer las demandas de la persona con discapacidad para así poder actuar consecuentemente. Consideran que el profesional, sea del ámbito sanitario, educativo o social, debe acompañar a la persona, y no dirigirla. Para ello debe ofrecerle la mayor cantidad de información posible que le permita llevar a cabo una decisión informada.

\section{De la presencia a la participación}

El debate en esta mesa se centró en hasta qué punto se ha pasado de la presencia de una manera natural de las personas con discapacidad en los contextos cotidianos, a una participación plena en dichos contextos, qué barreras están impidiendo este paso y qué líneas de actuación habría que desarrollar para eliminarlas.
En líneas generales se considera que aún queda mucho camino por recorrer, incluso hay una menor visibilidad de las asociaciones y un menor apoyo por parte de las instituciones. En el ámbito educativo la presencia se limita a unas determinadas discapacidades a las que a veces no se le puede dar el apoyo que contempla la legislación ... yo he hablado con gente que,... me han reconocido que la integración no existe, porque no hay medios, humanos, materiales... porque no hay. Si tú quieres matricular a tu hijo en un colegio ordinario va a ir a calentar una silla. Esta falta de apoyos deriva en el traslado a un colegio de educación especial, sin posibilidad además de elegir centro escolar.

Los profesores se encuentran con escasa formación, entrando así en juego la voluntariedad de formarse, y la actitud que tengan hacia la discapacidad, siendo este último aspecto crucial para potenciar o no la inclusión dentro del aula. Una de las ideas más repetidas y consensuadas es precisamente la actitud tiene que haber una buena actitud, primero, de los profesionales, y esos profesionales le tienen que transmitir esa buena actitud a los demás alumnos.

Entre las posibles barreras se reseña las llevadas a cabo incluso por los propios padres, sobreprotegiendo a la persona con discapacidad, y la existencia aún de barreras arquitectónicas a las que algunas veces se les busca una solución momentánea, como explicaba una persona con fibromialgia ante la situación en la que se encontró en un acto dirigido a personas con discapacidad cuando tenía que recoger un diploma en un escenario no adaptado me dijeron: se sube en brazos ¿verdad? en volandas hemos visto subir sillas de ruedas.

La mayoría del discurso global se centró en el cambio de actitud para avanzar en la participación. Esta actitud positiva hacia las capacidades de la persona, anteponiendo la cualidad de persona a la de discapacidad, debe adquirirse en las etapas más tempranas, en el ámbito educativo pero sin olvidar el ámbito social y familiar. Desde las instituciones deben lanzarse mensajes positivos a los padres sobre la presencia de personas con discapacidad en las aulas, cambiando el discurso de que la presencia entorpece a, que la necesidad de apoyos para la participación de una persona con discapacidad va en beneficio de todos puesto que no única y exclusivamente la persona con discapacidad se beneficia de ellos, estos suelen ser extensivos a quienes están a su alrededor.

Por otra parte se considera que la mera presencia no es suficiente, incluso puede ser dañina, 
como fue vivida por una persona con discapacidad. Yo analizando el proceso vivido con mi experiencia desde el 86, solo la presencia es perjudicial... porque todo el mundo lo ha traducido, las familias esto son problemas para mí, los profesionales, esto son problemas para mí y los papás de los niños afectados... Se indica la necesidad de desarrollar buenas prácticas profesionales y formar a las familias, de forma paralela a la presencia, para que se tengan los cimientos adecuados y se pueda progresar hacia la participación.

\section{Conclusiones}

A pesar de la exposición un tanto pesimista del estado actual de la discapacidad, consideran que en medio de la dificultad yace la oportunidad y que como han hecho en el pasado, es necesario remontar. Como decía un representante de una asociación somos gente pequeña, haciendo cosas pequeñas pero que al final moveremos el mundo, estoy seguro. De las ideas expuestas, avaladas muchas de ellas por investigaciones, incluso algunas recogidas por expertos muy recientemente ${ }^{14}$, se pueden extraer aspectos en las que hay que seguir avanzando.

El empoderamiento de las personas con discapacidad, reconocimiento de sus derechos y la consolidación de sociedades de coparticipación se encuentran con obstáculos como la crisis financiera y política, la globalización, la ausencia o debilidad de valores, la cultura de la inmediatez y la fragilidad del estado de bienestar ${ }^{15}$. Esta realidad conlleva a cambios en las organizaciones a partir de estrategias de denuncia y reivindicación. Se hace necesario organizaciones horizontales en vez de jerárquicas, enfocadas en la consecución de resultados personales, es decir buscando el beneficio en la calidad de vida, y alineadas con los investigadores y las políticas públicas. Las organizaciones no pueden seguir enraizadas en un modelo de expertos, asignando a la familia un mero papel de receptor de orientaciones ${ }^{14}$. Para ello la formación de los profesionales es crucial. Esta formación debe iniciarse en la infancia, mediante la incorporación activa de las personas con discapacidad en la sociedad, facilitando contextos donde muestren sus capacidades. Hay que prestar una especial atención a la formación de los futuros profesionales, teniendo como base en todas las titulaciones académicas una dedicación especial a la diversidad humana. Siguiendo las directrices del Espacio Europeo de Educación Superior, el futuro profesional debe ser capaz de asumir al menos los siguientes roles de aprendiz ${ }^{16}$ : aprendiz autónomo y estratégico, aprendiz en una comunidad, aprendiz de nuevas tecnologías; aprendiz dispuesto a asumir la movilidad y la diversidad, aprendiz creativo, crítico y reflexivo a las que,en el debate llevado a cabo, han añadido las competencias de saber compartir conocimientos y, escuchar y saber cómo dirigirse a la persona con discapacidad.

Igualmente se resalta la importancia de una coordinación entre los diferentes profesionales para llegar a un abordaje integral de la persona con discapacidad, de tal forma que la fusión de las actuaciones desde el ámbito sanitario, educativo y social consigan una calidad de vida que dé como resultado una inclusión plena de la persona.

Por otra parte, tras hacer una revisión en la base de datos europea Scopus se puede observar como la metodología cualitativa es cada vez más utilizada, siendo el área de la medicina donde se encuentran más escritos publicados y difundiéndose a nivel mundial. Se puede observar que el país con más cantidad de documentos divulgados, donde emplean este enfoque, es Estados Unidos, seguido de Reino Unido, Australia, Canadá y Brasil ${ }^{13,17}$.

Siguiendo el paradigma filosófico humanista, los enfoques cualitativos reconocen la singularidad del sujeto, el carácter intersubjetivo de la investigación empírica, y la solidaridad con los grupos encuestados, basándose en la igualdad fundamental del ser humano ${ }^{8}$. Bajo este supuesto filosófico, se ha optado en esta experiencia por una metodología narrativa a través de la utilización de entrevistas grupales y de la técnica de análisis de contenido. Mediante esta metodología, y de acuerdo con Sánchez Gómez et al. ${ }^{12}$, se tiene en cuenta la relevancia de los contextos, y por tanto, los procesos culturales; se parte del supuesto de que la realidad se construye socialmente, no es independiente de las personas; y se utiliza una técnica de recogida de datos sensible a nuevas realidades sociales, como son los grupos de discusión. 


\section{Colaboradores}

MV Martín Cilleros y MC Sánchez Gómez participaron en la concepción, interpretación de resultados y redacción, siendo responsables de la versión final del artículo. Además MV Martín Cilleros trabajó en la recogida y transcripción de datos. Además MC Sánchez Gómez trabajó en la metodología y análisis.

\section{Referencias}

1. Schalock R, Verdugo MA. A leadership guide for today's disabilities organizations: Overcoming challenges and making change happen. Baltimore: Brookes; 2012.

2. Gutiérrez-Ortega M, Martín-Cilleros MV, Jenaro-Río C. El Index para la inclusión: presencia, aprendizaje y participación. Rev nacional e internacional de educación inclusiva 2014; 7(3):186-201.

3. Gutiérrez-Ortega M. Evaluación de la inclusión en servicios para personas con discapacidad [tesis]. Salamanca: Universidad de Salamanca; 2011.

4. Verdugo MÁ, Gómez L, Navas P. Discapacidad e inclusión: derechos, apoyos y calidad de vida. In: Verdugo MA, Schalock R, organizadores. Discapacidad e inclusión. Manual para la docencia. Salamanca: Amarú; 2013. p. 17-41.

5. Celia Fiorati R, Meirelles V. Determinantes sociales de la salud, iniquidades e inclusión social entre personas con deficiencia. Rev. Latino-Am. Enfermagem 2015; 23(2):329-336.

6. Díaz Velázquez E. Ciudadanía, identidad y exclusión social de las personas con discapacidad. Politica y Sociedad 2010; 47(1):115-135.

7. Arias B, Verdugo MA, Gómez L, Arias V. Actitudes hacia la discapacidad. In: Verdugo MA, Schalock RL, organizadores. Discapacidad e Inclusión. Manual para la Docencia. Salamanca: Amarú; 2013. p. 61-88.

8. Minayo CS, Guerriero CZ. Reflexividade como éthos da pesquisa qualitativa. Cien Saude Colet 2014; 19(4):1103-1112.

9. Sánchez Gómez MC. Orígenes y evolución de la investigación de la investigación cualitativa en España. In: Souza N, Costa P. Investigação Qualitativa: Inovação, Dilemas e Desafios. Brasil: Ludomedia; 2015. p. 41-74.

10. Hernandez R, Fernández C, Baptista P. Metodología de Investigación. ciudad de Mexico: McGraw Hill; 2010.

\section{Agradecimientos}

Agradecemos a las personas que acudieron a la jornada su tiempo y dedicación, porque sin ellas no se hubiera podido realizar este estudio.

Igualmente agradecer al Servicio de Asuntos Sociales de la universidad de Salamanca su colaboración técnica y logística.

11. Milles MB, Huberman AM, Saldaña J. Qualitative data analysis: an expanded sourcebook. $3^{\text {rd }}$ ed. Thousand Oaks: Sage; 2013.

12. Sánchez Gómez MC, Delgado MC, Santos MC. El proceso de la investigación cualitativa. Manual de procedimiento:ejemplificación con una tesis doctoral Valladolid: Edintras; 2013.

13. Sánchez Gómez MC. La dicotomía cualitativo-cuantitativo: posibilidades de integración y diseños mixtos. Campo Abierto 2015; 1(1):11-30.

14. Giné C, Montero D, Verdugo MA, Rueda P, Vert S. Claves de futuro en la atención y apoyo a las personas con discapacidad intelectual y del desarrollo. ¿Qué nos dice la ciencia? Siglo Cero 2015; 46(1):81-106.

15. Lacasta J. Los vínculos entre el desarrollo humano y el desarrollo de las organizaciones. In Verdugo MA, Nieto T, Crespo M, Jordán de Urries B, organizadores. Cambio organizacional y apoyo a las graves afectaciones. Dos prioridades. Salamanca: Amarú; 2012. p. 79-113.

16. De Juanas Oliva A. Aprendices y competencias en el Espacio Europeo de Educación. Rev Psicología y Educación 2010; 5:171-186.

17. Sánchez Gómez MC, Martín Cilleros MV. Contextualización de la investigación cualitativa: de la confrontación al continuum. In: Conferencia Inaugural $1^{\circ} \mathrm{Con}$ gresso Educaçao, Pedagogía \& Innovaçao; 2015. Castelo Branco.

Artigo apresentado em 27/01/2016

Aprovado em 17/02/2016

Versão final apresentada em 19/02/2016 